International Journal of Research in Advent Technology, Vol.7, No.4, April 2019

E-ISSN: 2321-9637

Available online at www.ijrat.org

\title{
Teaching Plan Automation
}

\author{
Tanvi Umrani ${ }^{1}$, Mayuri Panchal ${ }^{2}$, Apurva Shirke ${ }^{3}$, Disha Shetty ${ }^{4}$ \\ BE, Department Of Computer Engineering ${ }^{1,2,3,4}$, Pimpri Chinchwad College of Engineering, Pune, India ${ }^{1,2,3,4}$ \\ Email:tanumrani@gmail.com ${ }^{1}$,mayuripanchal554@gmail.com ${ }^{2}$, shirkeapurva97@gmail.com ${ }^{3}$, \\ dkshetty1608@gmail.com ${ }^{4}$
}

\begin{abstract}
Traditional education system is dynamically changing to the concept of smart education, which faces many new challenges related to teaching session planning, management and monitoring. In this proposed system, we are providing the lecturer with a proper plan to manage and monitor the teaching sessions effortlessly. The traditional approach of creating a teaching session plan for teachers is a very hectic task and wastes a lot of time which could instead be used for some productive work. Our proposed system helps teachers to create Teaching Plan for generating the standardized Lesson Plans for every topic. Teaching Session Automation also helps the senior management of the colleges to keep a track of the Syllabus Status of each Class.
\end{abstract}

Keywords- Teaching-learning lesson plan, holiday calendar, syllabus, genetic algorithm, evolutionary algorithm, Genetic Artificial Immune Network, HTML, PHP, MYSQL, Yii, Wamp Server.

\section{INTRODUCTION}

Teachers spend the larger part of the day, providing answers to the essential questions, managing and organizing the students and work flow, creating the friendly learning ecosystem. The traditional approach of creating a teaching session plan for teachers is a very hectic task and wastes a lot of time which could instead be used for some productive work. Teaching Session Automation is a Web-based Software for Teachers and Colleges to Plan, Manage, and Track the Lesson Plans efficiently. This Lesson Plan Management System turns the tedious task of Lesson Planning into an absolutely remarkable experience for the teachers. The System helps teachers to create Lesson Plan Templates for designing the standardized Lesson Plans for every topic.

Teaching Session Automation is a seamless integration of Lesson Plan Management and Monitoring System; it also considers the holidays in the academic year which helps in managing and tracking the entire Academic Year's Teaching Plans. It helps the senior management of the colleges to keep a track of the Syllabus status of each Class.

\section{DIFFERENT APPROACHES}

The traditional approach of creating a teaching session planner for teachers is a very hectic task and wastes a lot of time which could instead be used for some productive work. Automated Teaching Session Planner System is a seamless integration of Time Table Management and Lesson Plan Management Software also considering the holidays in the academic year which helps in managing and tracking the entire Academic Year's Lesson Plans. It helps the senior management of the colleges to keep a track on the Syllabus Status of each Class. Our system provides the facility for Teachers to receive feedback for their Lesson Plans, and work towards its improvement. There are many different techniques to decrease teacher work load using technology. Automated Teaching Session Planner System is Web-based Software for Teachers and Colleges to Plan, Manage, and Track the Lesson Plans efficiently. Our
Automated teaching Session Planner System turns the tedious task of Lesson Planning into an absolutely remarkable experience for the teachers. Our System helps teachers to create Teaching Plan Templates for designing the standardized Lesson Plans for every topic.

In [1] Time Table creation Algorithm is main component of the project which produces the

HTML based time table even / odd semester sheet as the output. The project takes various inputs from the user such as Lecturer List, Topic List, Academic Calendar, Available room List, Day wise List and Time Chart. The knowledge base serves as input to the Timetable Generator Algorithm residing on server machine. The knowledge-base is in the middle, because it is between our timetabling algorithm and GUI front end which is designed in the last. After the representation of $\mathrm{KB}$ is standardized, they designed the time table generation algorithm. The Feature of security is strong so the record can be updated strictly only by Admin \& related faculty to that subject. The home page contains three categories of user. The specific login screen will appear on clicking any of the corresponding button. Password screen includes the username and password. On entering the valid credentials i.e. username and password then only the valid user is given access to the system. Logging in to the system can be done in three ways, as there are three users; that is, administrator login, students' login and faculties login. The admin here is responsible to register of the new student to the system.

In [2] provided solution is a website and it works as an alternative to the current timetable management system. We used Milestone approach as our research methodology. As the initial step, proper investigation could be launched about the current Time Table Generation System through interviewing the students, faculty and timetable in charge. As a result, advantages and disadvantages of that system could be clearly analyzed.

Then, in this paper they conducted an appropriate literature survey of their other works with referred to this subject to make an improvement in the problem 
International Journal of Research in Advent Technology, Vol.7, No.4, April 2019

E-ISSN: 2321-9637

Available online at www.ijrat.org

definition and find out technology to be used and its solution. Later, overall research design was constructed. Then, the system was developed using several tools such as PHP, MYSQL, Yii, Wamp Server and some more. Consequently, they implemented, deployed and evaluated that new system.

In [3] one user interface is created which, will be used by the valid administrator. This administrator is authorized to access the Database or the time table information of the institution. The same concept and services that is already used on the desktop for the purpose of time table maintenance is used on the palmtop. After creating the user interface then different forms are created. Some of the proposed forms are login-form, main-form etc. Linking of the database with the different created forms will be performed. Data Provider provides access to data source that is SQL server i.e. it provides object to achieve functionalities like opening and closing connection, retrieve data and update data. A Dataset represents a collection of Data Tables objects, together with the relationships and constraints that bind the various tables together. Data adapter acts as a bridge between data store and data set or in short it provides logic that would get data from the data source and populates the tables in the Dataset. Starting with debugging the application is deployed on the emulator. While deploying various emulation options are provided. The user can pick any of the emulator. Now the complete system will be made to run on the selected emulator. If the proposed system runs successfully on the emulator then only the application will be allowed to run on

any type of PDAs.

In [4] for solving timetable generation problem they have used evolutionary techniques. Algorithms like Genetic Algorithm, Evolutionary Algorithms were used. This paper centres on reviewing the difficulties of timetable generation in education system and using genetic algorithm to solve it. They have further used mimetic hybrid algorithm and Genetic Artificial Immune Network to solve this problem. Further they have compared the obtained solution with the solution obtained from genetic algorithm. By considering the result, they conclude that Genetic Artificial Immune Network can give optimal solution faster than that of genetic algorithm. track of the Syllabus status of each Class.

\section{PROPOSED SYSTEM}

In this system, we are providing an easy interface for the faculty to view their teaching plan. In this system, it takes faculty timetable as input which is further parsed and then the relevant data is stored in the database. On further processing, the data from database and form inputs, we get the total working days, from which we finally display the teaching plan

\subsection{Project modules}

There are two modules in teaching plan automation :

\subsubsection{Faculty Module}

Faculty members can login to the system through the portal with given credentials. Teachers can upload their faculty timetable and can track the coverage of topics. Teaching plan is provided to each faculty member after uploading faculty timetable.

\subsubsection{Admin Module}

Admin can login to the system through the portal with given credentials. Admin can upload the necessary files like holiday calendar, academic calendar and subject assigned to each faculty member. Admin can track the coverage of topics. from which we finally display the teaching plan

\subsection{Tools and technologies used}

\section{HTML:}

HTML is Hyper Text Markup Language.HTML is used to describe the structure of Web pages using markup. The elements of HTML are known as building blocks of HTML pages. Tags are used to represent HTML elements.

CSS:

CSS is Cascading Style Sheets.CSS is used to describe how HTML elements are to be displayed on screen, paper, or in other media.CSS can save a lot of work. Multiple web pages can be controlled by CSS all at once.

\section{Back End: Python, Django \\ Python}

Python is an interpreted, interactive, object-oriented, and high-level programming language. Python is designed to be readable language. Python has fewer syntactical constructions as compared to languages.

Django:Django is a web development framework which assists in building and maintaining quality webbased applications. It helps to eliminate repetitive tasks which makes the development process an easy and time saving experience.

\section{Database: MongoDB}

MongoDB is a popular NoSQL database language which stores data in flexible, JSON-like documents, meaning fields can vary from document to document and data structure can be changed over time. The document model maps to the objects in your application code, making data easy to work with Ad hoc queries, indexing, and real time aggregation provide powerful ways to access and analyze your data. MongoDB is a distributed database at its core, so high availability, horizontal scaling, and geographic distribution are built in and easy to use MongoDB is free and open-source, published under the GNU Affero General Public License.

\section{PyCharm:}

PyCharm is an integrated development environment (IDE) used in computer programming for the Python language. $t$ provides code analysis, a graphical debugger, an integrated unit tester, integration with version control systems (VCSes), and supports web development with Django. 


\subsection{System Architecture}

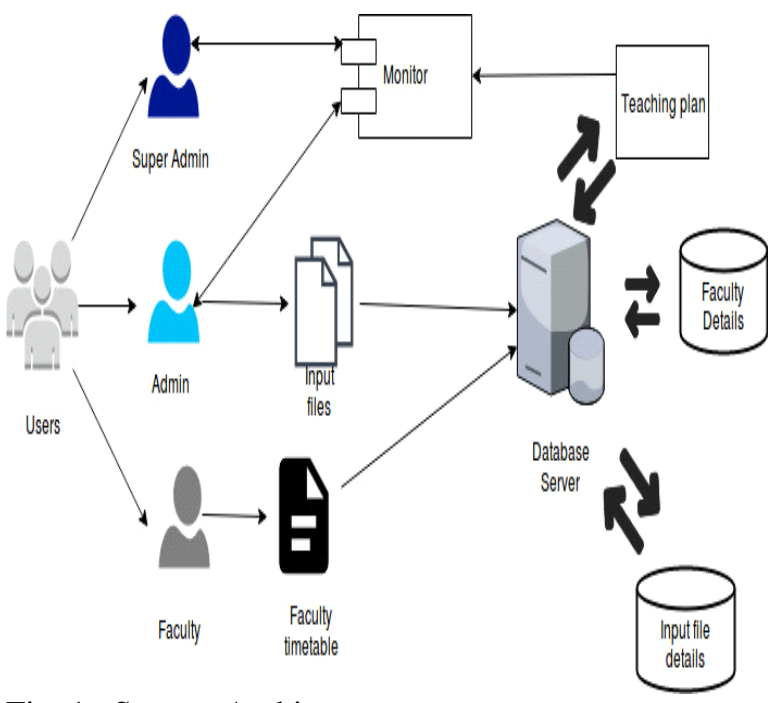

Fig. 1. System Architecture

\subsection{Algorithm}

\#Open and read input files

File1 = open () \#File1

File2 $=$ open ()$\#$ File2

File3 = open () \#File3

\#Extracting data from files and storing in database

Function process (File1, File2, File3)

\{

$\mathrm{X}=$ processAcademicCalendar (File1)

$\mathrm{Y}=$ processHolidayCalendar (File2)

$\mathrm{Z}=$ processLecturerTimetable (File 3 )

\}

\#Retrieve data stored from database to create automated teaching plan

Function createTeachingPlan (X, Y, Z)

\{

1. Calculate the working days

Working days $=($ startdate-endSemdate $)$-Holidays

2. Calculate teaching load

3. Generate teaching plan accordingly

$$
\text { \} }
$$

\section{CONCLUSION}

This survey has been performed for gathering the details of Teaching plan Automation system and to find out different effective methods which are useful for generating teaching plan for faculties. Separate Teaching plan for the individual faculty is generated automatically by this system. The system reduces time consumption and pain in framing the teaching plan manually. The system is useful to automate the academic process which helps in reducing complex work.

\section{REFERENCES}

[1] Anuja Chowdhary, Priyanka Kakde, Shruti Dhoke, Sonali Ingle, Rupal Rushiya, Dinesh Gawande, International Journal of Computer Science and Mobile Computing, Vol.3 Issue.2, February- 2014, pg. 410-414.

[2] Chaya Andradi, Saminda Premaratne (Faculty of IT, University of Moratuwa, Sri Lanka), International Journal of Modern Research in Engineering and Technology (IJMRET) www.ijmret.org Volume 1 Issue $2 \|$ July 2016.

[3] Jitendra V. Tembhurne (Department of ComputerTechnology, Kavikulguru Institute of Technology \& Science,Ramtek, Nagpur,Maharashtra,India email:jitendratembhurne@gmail.com),Dilipkumar A. Borikar (Department of Information Technology, Kavikulguru Institute of Technology \& Science, Ramtek, Nagpur, Maharashtra, India.

[4] Bhaduri a "university timetable scheduling using genetic algorithm". Advances in Recent Technologies in Communication and Computing, 2009. ARTCom '09. International Conference 